\title{
Some Transformation of a Multiple Hypergeometric Series of Lauricella Function of $\boldsymbol{n}$ Variables
}

Nabiullah Khan ${ }^{*}$ and T. Kashmin

Department of Applied Mathematics, Faculty of Engineering and Technology, Aligarh Muslim University, Aligarh 202002, India

Abstract: This paper deals with an integral transformation involving Whittaker function $M_{k, m}(x)$ into a multiple hypergeometric series of Lauricella function $F_{A}^{(n)}$ of $n$ variables. A number of known and new transformation and reduction formulae for a hypergeometric function ${ }_{2} F_{1}$, Appell function $F_{2}$, Lauricella function $F_{A}^{(3)}$ and a hypergeometric function of four variables $F_{p}^{(4)}$ are derived as special cases.

Key words: Whittaker function, Lauricella function, Laplace transform.

\section{Introduction and Definition}

Lauricella [1] further generalized the four Appell functions $F_{1}, \ldots, F_{4}$ to functions of $n$-variables and defined his functions as follows [2]:

$$
\begin{gathered}
F_{A}^{(n)}\left[a, b_{1}, \ldots, b_{n} ; c_{1}, \ldots, c_{n} ; x_{1}, \ldots, x_{n}\right] \\
=\sum_{m_{1}, \ldots, m_{n}=0}^{\infty} \frac{(a)_{m_{1}+\cdots+m_{n}}\left(b_{1}\right)_{m_{1}} \ldots\left(b_{n}\right)_{m_{n}}}{\left(c_{1}\right)_{m_{1}} \ldots\left(c_{n}\right)_{m_{n}}} \frac{x_{1}^{m_{1}}}{m_{1} !} \ldots \frac{x_{n}^{m_{n}}}{m_{n} !} \\
\left|x_{1}\right|+\cdots+\left|x_{n}\right|<1 ;
\end{gathered}
$$

Some transformations of Lauricella function which is given by Srivastava H.M and Manocha H.L [2]:

$$
F_{A}^{(1)}={ }_{2} F_{1}, F_{A}^{(2)}=F_{2}
$$

where, ${ }_{2} F_{1}, F_{2}$ are Gauss hypergeometric function and Appell function

A general triple hypergeometric series $F^{(3)}$ of Srivastava's [2] defined as:

$$
\begin{aligned}
F^{(3)}\left[\begin{array}{l}
(a)::(b) ;\left(b^{\prime}\right) ;\left(b^{\prime \prime}\right) ;(e) ;\left(e^{\prime}\right) ;\left(e^{\prime \prime}\right) ; \\
(d)::(g) ;\left(g^{\prime}\right) ;\left(g^{\prime \prime}\right) ;(h) ;\left(h^{\prime}\right) ;\left(h^{\prime \prime}\right) ;
\end{array}\right] \\
=\sum_{m, n, p=0}^{\infty} \frac{[(a)]_{m+n+p}[(b)]_{m+n}\left[\left(b^{\prime}\right)\right]_{n+p}\left[\left(b^{\prime \prime}\right)\right]_{p+m}[(e)]_{m}\left[\left(e^{\prime}\right)\right]_{n}\left[\left(e^{\prime \prime}\right)\right]_{p}}{[(d)]_{m+n+p}[(g)]_{m+n}\left[\left(g^{\prime}\right)\right]_{n+p}\left[\left(g^{\prime \prime}\right)\right]_{p+m}[(h)]_{m}\left[\left(h^{\prime}\right)\right]_{n}\left[\left(h^{\prime \prime}\right)\right]_{p}} \frac{x^{m}}{m !} \frac{y^{n}}{n !} \frac{z^{p}}{p !}
\end{aligned}
$$

where, (a) means $a_{1}, a_{2}, \ldots a_{n}$ and $(a)_{n}$ has the interpretation

$$
\prod_{j=1}^{A}\left(a_{j}\right)_{n}=\prod_{j=1}^{A} \frac{\left.\Gamma a_{j}+n\right)}{\Gamma\left(a_{j}\right)}
$$

A hypergeometric function of four variables defined by Pathan [3] are given in the following form:

\footnotetext{
*Corresponding author: Nabiullah Khan, Associate Professor, research field: special function. E-mail: nabi_khan1@rediffmail.com.
} 


$$
\begin{gathered}
F_{p}^{(4)}\left[\begin{array}{l}
a:: b ;-;-;-; d ; e ; f, g ; \\
c::-;-;-;-; d^{\prime} ; e^{\prime} ; f^{\prime} ; \quad u, y, z
\end{array}\right] \\
=\sum_{m, n, p, q=0}^{\infty} \frac{(a)_{m+n+p+q}(b)_{m+n+p}(d)_{m}(e)_{n}(f)_{p}(g)_{q}}{(c)_{m+n+p+q}\left(d^{\prime}\right)_{m}\left(e^{\prime}\right)_{n}\left(f^{\prime}\right)_{p}} \frac{u^{m}}{m !} \frac{x^{m}}{n !} \frac{y^{n}}{p !} \frac{z^{p}}{q !}
\end{gathered}
$$

\section{Main Transformation}

An integral is established in the following form:

$$
\begin{gathered}
\int_{0}^{\infty} t^{\lambda-1} e^{-\left(z+\frac{p}{2}\right) t} M_{k, \mu}(p t) M_{k_{1}, v_{1}-\frac{1}{2}}\left(y_{1} t\right) \ldots \ldots M_{k_{n}, v_{n}-\frac{1}{2}}\left(y_{n} t\right) d t \\
=\frac{p^{\mu+\frac{1}{2}} y_{1}^{v_{1}} \ldots \ldots y_{n}^{v_{n}} \Gamma(b+r)}{\tau^{(b+r)}} \sum_{r=0}^{\infty} \frac{\left(\frac{1}{2}+\mu-k\right)_{r} p^{r}}{(2 \mu+1)_{r} r !} \\
F_{A}^{(n)}\left[b+r ; v_{1}-k_{1}, \ldots, v_{n}-k_{n} ; 2 v_{1}, \ldots, 2 v_{n} ; \frac{y_{1}}{\tau}, \ldots ., \frac{y_{n}}{\tau}\right]
\end{gathered}
$$

where, $b=\lambda+\mu+v_{1}+\cdots \ldots . .+v_{n}+\frac{1}{2}, \operatorname{Re}\left(z+p+\frac{1}{2} Y\right)>0, Y=y_{1}+\cdots \ldots+y_{n}, \operatorname{Re}(b+r)>0$,

$\tau=z+p+\frac{1}{2} Y$.

$F_{A}^{(n)}$ is called Lauricella function defined by Eq. (1) and $M_{k, m}(x)$ is Whittaker function [4, 5] defined as:

$$
M_{k, m}(x)=x^{1 / 2+m} e^{-1 / 2 x} F_{1}\left(1 / 2+m-k, 2 m_{1}+1 ; x\right)=x^{1 / 2+m} e^{-1 / 2 x} \sum_{q=0}^{\infty} \frac{\left(\frac{1}{2}+m-k\right)_{q} x^{q}}{(2 m+1)_{q} q !}
$$

Eq. (5) can be obtained by expanding $M_{k, m}(x)$ in series with the help of Eq. (6) and integrating term by term with the help of the following result [6]:

$$
\begin{gathered}
\int_{0}^{\infty} t^{v-1} e^{-p t} M_{k_{1}, \mu_{1}-\frac{1}{2}}\left(a_{1} t\right) \ldots \ldots M_{k_{n}, \mu_{n}-\frac{1}{2}}\left(a_{n} t\right) d t=\frac{a_{1}^{\mu_{1}} \ldots \ldots a_{n}^{\mu_{n}} \Gamma(v+M)}{(p+A)^{(v+M)}} \\
F_{A}^{(n)}\left[v+M ; \mu_{1}-k_{1}, \ldots ., \mu_{n}-k_{n} ; 2 \mu_{1}, \ldots, 2 \mu_{n} ; \frac{a_{1}}{(p+A)}, \ldots ., \frac{a_{n}}{(p+A)}\right]
\end{gathered}
$$

where, $M=\mu_{1}+\ldots . .+\mu_{n}, A=1 / 2\left(a_{1}+\ldots . . a_{n}\right), \operatorname{Re}(v+M)>0, \operatorname{Re}\left(p \pm 1 / 2 a_{1}+\ldots \ldots \pm 1 / 2 a_{n}\right)>0$.

\section{Special Cases}

Some special cases of the main result Eq. (5) are given below:

(1) Setting $n=1$ in Eq. (5), we get a result associated with Eq. (2):

$$
\begin{gathered}
\int_{0}^{\infty} t^{\lambda-1} e^{-\left(z+\frac{p}{2}\right) t} M_{k, \mu}(p t) M_{k_{1}, v_{1}-\frac{1}{2}}\left(y_{1} t\right) d t=\frac{p^{\mu+\frac{1}{2}} y_{1}^{v_{1}} \Gamma\left(\lambda+\mu+v_{1}+\frac{1}{2}+r\right)}{\left(z+p+\frac{y_{1}}{2}\right)^{\left(\lambda+\mu+v_{1}+\frac{1}{2}+r\right)}} \sum_{r=0}^{\infty} \frac{\left(\frac{1}{2}+\mu-k\right)_{r} p^{r}}{(2 \mu+1)_{r} r !} \\
\quad \times_{2} F_{1}\left(\begin{array}{l}
\lambda+\mu+v_{1}+\frac{1}{2}+r, v_{1}-k_{1} ; \\
2 v_{1}
\end{array} \quad ; \quad \frac{y_{1}}{\left(z+p+\frac{y_{1}}{2}\right)}\right)
\end{gathered}
$$


where, $\operatorname{Re}\left(\lambda+\mu+v_{1}+\frac{1}{2}\right)>0, \operatorname{Re}\left(z+p+\frac{y_{1}}{2}\right)>0$

(2) Setting $n=2$ in Eq. (5) and using Eq. (2), we get Appell function $F_{2}$ :

$$
\begin{gathered}
\int_{0}^{\infty} t^{\lambda-1} e^{-\left(z+\frac{p}{2}\right) t} M_{k, \mu}(p t) M_{k_{1}, v_{1}-\frac{1}{2}}\left(y_{1} t\right) M_{k_{2}, v_{2}-\frac{1}{2}}\left(y_{2} t\right) d t=\frac{p^{\mu+\frac{1}{2}} y_{1}^{v_{1}} y_{2}^{v_{2}} \Gamma(b+r)}{\left(z+p+\frac{y_{1}}{2}+\frac{y_{2}}{2}\right)^{b+r}} \sum_{r=0}^{\infty} \frac{\left(\frac{1}{2}+\mu-k\right)_{r} p^{r}}{(2 \mu+1)_{r} r !} \\
\times F_{2}\left[b+r ; v_{1}-k_{1}, v_{2}-k_{2} ; 2 v_{1}, 2 v_{2} ; \frac{y_{1}}{\left(z+p+\frac{y_{1}}{2}+\frac{y_{2}}{2}\right)}, \frac{y_{2}}{\left(z+p+\frac{y_{1}}{2}+\frac{y_{2}}{2}\right)}\right]
\end{gathered}
$$

where, $b=\lambda+\mu+v_{1}+v_{2}+\frac{1}{2}, \operatorname{Re}\left(z+p+\frac{y_{1}}{2}+\frac{y_{2}}{2}\right)>0, \operatorname{Re}(b+r)>0$

Appell's function $F_{2}[2]$ is defined as:

$$
F_{2}\left[a, b, b^{\prime} ; c, c^{\prime} ; x, y\right]=\sum_{m, n=0}^{\infty} \frac{(a)_{m+n}(b)_{m}\left(b^{\prime}\right) n x^{m} y^{n}}{(c)_{m}\left(c^{\prime}\right)_{n} m ! n !}
$$

(3) Setting $k_{1}=k_{2}=0$ in Eq. (9) and using the following known transformation [7]:

$$
\begin{gathered}
F_{2}\left[\alpha, \beta, \beta^{\prime}, 2 \beta, 2 \beta^{\prime} ; 2 x, y\right]=(1-x)^{-\alpha} H_{4}\left[\alpha, \beta^{\prime}, \beta+\frac{1}{2}, 2 \beta^{\prime} ; \frac{x^{2}}{4(1-x)^{2}}, \frac{y}{1-x}\right] \\
\int_{0}^{\infty} t^{\lambda-1} e^{-\left(z+\frac{p}{2}\right) t} M_{k, \mu}(p t) M_{0, v_{1}-\frac{1}{2}}\left(y_{1} t\right) M_{0, v_{2}-\frac{1}{2}}\left(y_{2} t\right) d t=\frac{p^{\mu+\frac{1}{2}} y_{1}^{v_{1}} y_{2}^{v_{2}} \Gamma(b+r)}{\left(z+p+\frac{y_{1}}{2}+\frac{y_{2}}{2}\right)^{b+r}} \sum_{r=0}^{\infty} \frac{\left(\frac{1}{2}+\mu-k\right)_{r} p^{r}}{(2 \mu+1)_{r} r !} \\
\times F_{2}\left[b+r, v_{1}, v_{2} ; 2 v_{1}, 2 v_{2} ; \frac{y_{1}}{\left(z+p+\frac{y_{1}}{2}+\frac{y_{2}}{2}\right)}, \frac{y_{2}}{\left(z+p+\frac{y_{1}}{2}+\frac{y_{2}}{2}\right)}\right]
\end{gathered}
$$

We get an infinite sum of Horn function $H_{4}$ in the following form:

$$
\begin{gathered}
\int_{0}^{\infty} t^{\lambda-1} e^{-\left(z+\frac{p}{2}\right) t} M_{k, \mu}(p t) M_{0, v_{1}-\frac{1}{2}}\left(y_{1} t\right) M_{0, v_{2}-\frac{1}{2}}\left(y_{2} t\right) d t=p^{\mu+\frac{1}{2}} y_{1}^{v_{1}} y_{2}^{v_{2}} \Gamma(b+r) \sum_{r=0}^{\infty} \frac{\left(\frac{1}{2}+\mu-k\right)_{r} p^{r}}{(2 \mu+1)_{r} r !} \\
\times\left(z+p-\frac{3 y_{1}}{2}+\frac{y_{2}}{2}\right)^{-b-r} H_{4}\left[b+r, v_{2}, v_{1}+\frac{1}{2}, 2 v_{2} ; \frac{y_{1}^{2}}{\left(z+p-\frac{y_{1}}{2}+\frac{y_{2}}{2}\right)^{2}}, \frac{y_{2}}{\left(z+p-\frac{3 y_{1}}{2}+\frac{y_{2}}{2}\right)}\right]
\end{gathered}
$$

where, $H_{4}$ is the Horn function [8] defined as:

$$
H_{4}[\alpha, \beta, \gamma, \delta ; x, y]=\sum_{m, n=0}^{\infty} \frac{(\alpha)_{2 m+n}(\beta)_{n} x^{m} y^{n}}{(\gamma)_{m}(\delta)_{n} m ! n !}
$$

(4) Setting $k_{1}=k_{2}=0$ in Eq. (9) and using the following result of Srivastava and Karlsson [9]

$$
F_{2}=F_{0: 1: 1}^{1: 1}
$$

we get a Kampe $e^{\prime}$ de Fe'riet function of two variables:

$$
\begin{gathered}
\int_{0}^{\infty} t^{\lambda-1} e^{-\left(z+\frac{p}{2}\right) t} M_{k, \mu}(p t) M_{0, v_{1}-\frac{1}{2}}\left(y_{1} t\right) M_{0, v_{2}-\frac{1}{2}}\left(y_{2} t\right) d t=\frac{p^{\mu+\frac{1}{2}} y_{1}^{v_{1}} y_{2}^{v_{2}} \Gamma(b+r)}{\tau^{b+r}} \sum_{r=0}^{\infty} \frac{\left(\frac{1}{2}+\mu-k\right)_{r} p^{r}}{(2 \mu+1)_{r} r !} \\
F_{0: 1: 1}^{1: 1: 1}\left[\begin{array}{ll}
b+r ; v_{1}, v_{2} ; & \frac{y_{1}}{\tau}, \frac{y_{2}}{\tau} \\
: 2 v_{1}, 2 v_{2} ; &
\end{array}\right.
\end{gathered}
$$

where, $b=\lambda+\mu+v_{1}+v_{2}+\frac{1}{2}, \tau=z+p+\frac{y_{1}}{2}+\frac{y_{2}}{2}$ and $F_{l: m: n}^{p: q: s}$ is Kamp $e^{\prime}$ de $\mathrm{F} e^{\prime}$ riet function defined by Srivastava and Panda [10]. 
(5) Setting $n=3$ in Eq. (5), we get a new result associated with Lauricella's function $F_{A}^{(3)}$ as:

$$
\begin{gathered}
\int_{0}^{\infty} t^{\lambda-1} e^{-\left(z+\frac{p}{2}\right) t} M_{k, \mu}(p t) M_{k_{1}, v_{1}-\frac{1}{2}}\left(y_{1} t\right) M_{k_{2}, v_{2}-\frac{1}{2}}\left(y_{2} t\right) M_{k_{3}, v_{3}-\frac{1}{2}}\left(y_{3} t\right) d t \\
=\frac{p^{\mu+\frac{1}{2}} y_{1}^{v_{1}} y_{2}^{v_{2}} y_{3}^{v_{3}} \Gamma(b+r)}{\tau^{b+r}} \sum_{r=0}^{\infty} \frac{\left(\frac{1}{2}+\mu-k\right)_{r} p^{r}}{(2 \mu+1)_{r} r !} \\
\times F_{A}^{(3)}\left[b+r ; v_{1}-k_{1}, v_{2}-k_{2}, v_{3}-k_{3} ; 2 v_{1}, 2 v_{2}, 2 v_{3} ; \frac{y_{1}}{\tau}, \frac{y_{2}}{\tau}, \frac{y_{3}}{\tau}\right]
\end{gathered}
$$

where, $b=\lambda+\mu+v_{1}+v_{2}+v_{3}+\frac{1}{2}, \operatorname{Re}(b+r)>0, \tau=z+p+\frac{y_{1}+y_{2}+y_{3}}{2}, \operatorname{Re}\left(z+p+\frac{y_{1}+y_{2}+y_{3}}{2}\right)>0$.

Using a following known result of Srivastava and Karlsson [9]:

$$
\begin{aligned}
& F_{A}^{(3)}\left[\alpha, \beta_{1}, \beta_{2}, \beta_{3} ; \gamma_{1}, \gamma_{2}, \gamma_{3} ; x, y, z\right] \\
& \quad=F^{(3)}\left[\begin{array}{l}
(\alpha):: \_; \_; \_; \beta_{1}, \beta_{2}, \beta_{3} ; \\
: \because \_; \_; \_; \gamma_{1}, \gamma_{2}, \gamma_{3} ;
\end{array}\right]
\end{aligned}
$$

the Eq. (17) becomes:

$$
\begin{gathered}
\int_{0}^{\infty} t^{\lambda-1} e^{-\left(z+\frac{p}{2}\right) t} M_{k, \mu}(p t) M_{k_{1}, v_{1}-\frac{1}{2}}\left(y_{1} t\right) M_{k_{2}, v_{2}-\frac{1}{2}}\left(y_{2} t\right) M_{k_{3}, v_{3}-\frac{1}{2}}\left(y_{3} t\right) d t \\
=\frac{p^{\mu+\frac{1}{2}} y_{1}^{v_{1}} y_{2}^{v_{2}} y_{3}^{v_{3}} \Gamma(b+r)}{\tau^{b+r}} \sum_{r=0}^{\infty} \frac{\left(\frac{1}{2}+\mu-k\right)_{r} p^{r}}{(2 \mu+1)_{r} r !} \\
F^{(3)}\left[\begin{array}{l}
b+r::_{-} ; \ldots ;-; \\
{\left[v_{1}-k_{1}, v_{2}-k_{2}, v_{3}-k_{3} ; \quad \frac{y_{1}}{\tau}, \frac{y_{2}}{\tau}, \frac{y_{3}}{\tau}\right.}
\end{array}\right]
\end{gathered}
$$

where, $b=\lambda+\mu+v_{1}+v_{2}+v_{3}+\frac{1}{2}, \operatorname{Re}(b+r)>0, \tau=z+p+\frac{y_{1}+y_{2}+y_{3}}{2}, \operatorname{Re}\left(z+p+\frac{y_{1}+y_{2}+y_{3}}{2}\right)>0 . F^{(3)}$ is the triple hypergeometric series defied by Eq. (3).

Further expanding $F^{(3)}$ into a series with the help of Eq. (3), we get a hypergeometric function $F_{p}^{(4)}$ of four variables defined by Pathan [7]:

$$
\begin{aligned}
& \int_{0}^{\infty} t^{\lambda-1} e^{-\left(z+\frac{p}{2}\right) t} M_{k, \mu}(p t) M_{k_{1}, v_{1}-\frac{1}{2}}\left(y_{1} t\right) M_{k_{2}, v_{2}-\frac{1}{2}}\left(y_{2} t\right) M_{k_{3}, v_{3}-\frac{1}{2}}\left(y_{3} t\right) d t=\frac{p^{\mu+\frac{1}{2}} y_{1}^{\nu_{1}} y_{2}^{v_{2}} y_{3}^{\nu_{3}} \Gamma(b)}{\tau^{b}}
\end{aligned}
$$

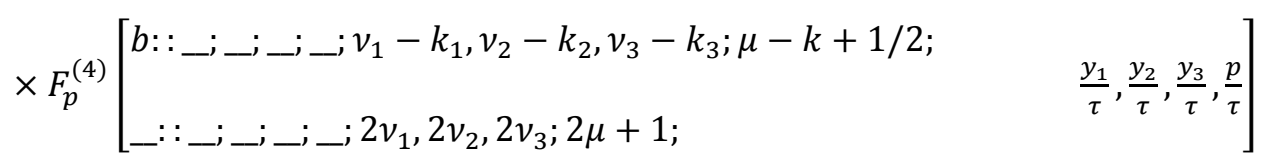

If we replace Whittaker function $M_{k, \mu}(p t)$ by $W_{k, \mu}(p t)$ in Eq. (20), we will get a known result of Pathan [7].

(6) Setting $k=k_{1}=k_{2}=k_{3}=0=\lambda, z=\frac{-p}{2}, v_{1}=\mu+\frac{1}{2}, v_{2}=v_{3}=v+\frac{1}{2}, p=2 \alpha, y_{1}=2 \beta, y_{2}=2 \gamma, y_{3}=$ $2 \delta$ in Eq. (20) and using the following known result [4]

we get

$$
M_{0, \mu}(x)=2^{2 \mu} \Gamma(\mu+1) x^{\frac{1}{2}} I_{\mu}\left(\frac{1}{2} x\right)
$$




$$
\begin{aligned}
& \int_{0}^{\infty} t I_{\nu}(\alpha t) I_{\nu}(\beta t) I_{\mu}(\gamma t) I_{\mu}(\delta t) d t \\
& =\frac{(\alpha \beta)^{\mu}(\gamma \delta)^{v} \rho^{-2-2 \mu-2 v} \Gamma(2+2 \mu+2 v)}{2^{2 \mu+2 v}(\Gamma(\mu+1))^{2}(\Gamma(v+1))^{2}}
\end{aligned}
$$

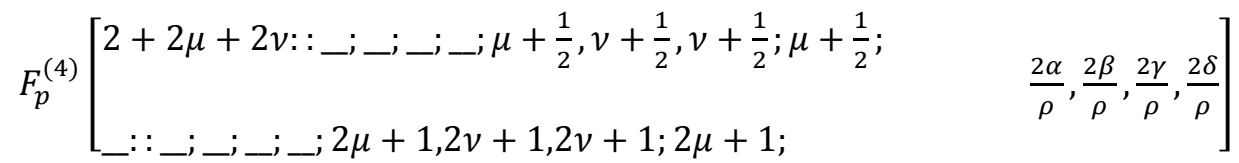

where, $\rho=\alpha+\beta+\gamma+\delta, \operatorname{Re}(1+\mu+v)>0$ and $\operatorname{Re}(\alpha+\beta+\gamma+\delta)>0$. Eq. (21) gives an interesting new result for the hypergeometric function $F_{p}^{(4)}$ and $I_{v}(x)$ is the modified Bessel function [2; p. 209(1)] defined as

$$
I_{v}(x)=\frac{(x / 2)^{v}}{\Gamma(v+1)}{ }_{0} F_{1}\left[\begin{array}{l}
-; \\
v+1 ;
\end{array} \quad \frac{x^{2}}{4}\right]
$$

\section{Conclusions}

In the present investigation, we have established an integral transformation involving Whittaker function $M_{k, m}(x)$ into a multiple hypergeometric series of Lauricella function $F_{A}^{(n)}$ of $n$ variables, and generalize a number of known and new transformation and reduction formulae for a hypergeometric function ${ }_{2} F_{1}$, Appell function $F_{2}$, Lauricella function $F_{A}^{(3)}$ and a hypergeometric function of four variables $F_{p}^{(4)}$. The results appear in this paper may be found useful in some areas of mathematical physics and Engineering.

\section{Acknowledgements}

The authors are thankful to professor Pathan M.A of Aligarh Muslim University, India for his valuable suggestions.

\section{References}

[1] Lauricella, G. 1893. "Sulle Funzioni Ipergeometriche a Piu Variabili." Rend. Circ. Mat. Palermo 7: 111-158.

[2] Srivastava, H. M., and Manocha, H. L. 1984. A Treatise on Generating Functions. Halsted Press (Ellis Horwood Limited, Chichester): John Wiley and sons, New York.

[3] Pathan, M. A. 1979. "On a Transformation of a General Hypergeometric Series of Four Variables.” Nederl, Akad. Wetensch. Proc Ser. A. 82=Indag Math $\underline{41}$, New York, 171-5.

[4] Whittaker, E. T. 1903. "An Expression of Certain Known Function as Generalized Hypergeometric Functions.” Bull. Amer. Math. Soc. 10: 125-134.

[5] Whittaker, E. T., and Watson, G. N. 1990. A Course of Modern Analysis, 4th ed. Cambridge, England.Cambridge University.

[6] Erdelyi, A., 1954. "Table of integral transforms." Vol.I, McGraw-Hill, New York.

[7] Erdelyi, A. 1948. "Transformation of Hypergeometric Functions of Two Variables." proc. Roy, Soc. Edinburgh Sect. A 62: 378-385.

[8] Khan, B., and Pathan, M. A. 1982. "Some Transformations of Appell's Function $\mathrm{F}_{4}$." Tamkang $J$. Math. 13 (1): 25-35.

[9] Srivastav, H. M., and Karlsson, P. W. 1985. Multiple Gaussian Hypergeometric Series. Halsted Press (Ellis Horwood Limited, Chichester): John Wiley and sons, New York, Chichester Brisbane, Toronto.

[10] Srivastav, H. M., and Panda, R. 1976. "An Integral Representation for the Product of Two Jacobi Polynomials.” J. London Math. Soc. 12 (2): 419-425. 